\title{
Editorial Translational Myology for Physical Medicine and Rehabilitation: the evidence based approach
}

Evidence for the effectiveness of physical therapy modalities to improve mobility functional performances, muscle characteristics and pain management become more important, particularly when social security institutions discovered Evidence Based Medicine (EBM) as a policy for financial compensation of therapies and therapy guidelines. In believe of EBM social security institutions more often use scientific "external evidence" neglecting "individual clinical expertise" and "patient values" as Sackett et al. and the Cochrane Collaboration proposed. Sackett, a founder of EBM published in 1996 how three pillars are building the value of therapies, but that use of EBM may raise rather than lower the costs. However, even if no strong evidence exists from randomized controlled trials for a treatment/method, the next levels should be considered too. In no case, however, the clinical experience and the patient's value should be forgotten as key decision criteria! Finally, referring to Sackett, it is the treating physician who rates the importance of scientific literature for the individualized treatment of a patient.

This issue highlights different aspects of scientific studies conducted to increase knowledge of muscle physiology and molecular biology. What they have in common is the "evidence based approach" and the impact as translational medicine for Physical Medicine and Rehabilitation. Gerta Vrbová gives a brief introduction to the historic researches of AV Hill, which greatly contributed to our todays understanding of muscle contraction. His discoveries helped solving the problem of how chemical events provide the energy for mechanical work of human muscle. Hill helped to train and educate a generation of scientists to use concise mathematical treatment of biological phenomena, the strong grounds of EBM. The sarcomeric cytoskeleton and its L-type $\mathrm{Ca} 2+$ channels that regulate the $\mathrm{Ca} 2+$ entry during mechanical stretch of the muscle fibers are rewieved by Fabio Francini and Roberta Squecco. Taken together with the electron microscopic findings of Anna Jakubiec-Puka on effects of passive and active stretching, they provide the translational basis for our clinical work. They, indeed, explaining why complete denervated or incomplete paralysed human patients may benefit from physical therapy, represent sound examples of Translational Medicine. In children with plexus pareses, conus cauda patients and patients with peripheral nerve lesions we may successfully counteract the ongoing atrophy process of denervated / plegic muscle fibers with passive movements. The treatment activates the calcium influx by passive movements up to 200 or 300 repetitions per day in series of 20-30 repetitions, ten times a day for all paralysed muscles groups.

The contributions of Milan Sedliak et al. and Nejc Sarabon et al. are focused on the neuromuscular control in elderly people. The first group developed a novel test for postural sway measurement and investigated its reliability, whereas the latter group examined the impact of strength training on static balance as a lower extremity postural task test. The improved ability of seniors has an important influence in protecting them from falling and from the potential consequences, like fractures of the hip, femoral neck, pelvis and legs.

A technical contribution regarding data transmission with wireless implants from Lukas Kneisz et al. deals with the influence of insulation layers of the transmitter antennas. Authors, mimicking at the bench the detuning effects of biological tissues and concluding that antennas coated with tissue equivalent material provides a safer transmission of recorded data from inside the body to external receivers, provide hard data and important developments for future implantable functional devices and technical aids in Physical Medicine and Rehabilitation. A further example of the evidence based approach in Physical Medicine and Rehabilitation is from Asami Naka et al. Reviewing the literature on analgesic effects of TENS in different pain conditions between 2007 and 2012, they show that TENS has an analgesic effect in various pain conditions and is therefore useful in clinical practice. Functional and structural muscle decline is a major problem during aging. Our goal was to improve in old subjects quadriceps $\mathrm{m}$. force and mobility functional performances by FES. By histological and biological molecular analyses of vastus lateralis m. biopsies, we demonstrate that electrical stimulation significantly improves mobility performances, muscle structural characteristics and molecular markers in 70-year old seniors.

Sackett DL, Rosenberg WM, Gray JA, Haynes RB, Richardson WS. Evidence based medicine: what it is and what it isn't. BMJ. 1996; 312(7023): 71-72.

Jakubiec-Puka A, Sławińska U, Rędowicz MJ, et al. Beneficial effect of locomotor training on the structure of the denervated rat soleus muscle. Basic Appl Myol 2007; 17: 125-127.

Helmut Kern, MD, PhD, Editor - Translational Myology

Institute for Physical Medicine and Rehabilitation, Wilhelminenspital and

Ludwig Boltzmann Institute of Electrical Stimulation and Physical Rehabilitation

Montleartstrasse 37, 1160 Vienna, Austria

E-mail: wil.pys.kern-forschung@wienkav.at 


\section{Editorial Translationale Myologie für Physikalische Medizin und Rehabilitation: Ein evidenzbasierter Ansatz}

Der Wirkungsnachweis physikalischer Behandlungsmethoden zur Verbesserung der Funktion, der muskulären Eigenschaften und zur Schmerztherapie ist besonders bedeutungsvoll und umso wichtiger je mehr die Sozialversicherungsträger die evidenzbasierte Medizin (EBM) als oberste Richtlinie für die finanzielle Abgeltung von Therapien und für Therapierichtlinien heranziehen. Im Bereich der EBM wird jedoch von den Sozialversicherungsträgern oft der Fehler gemacht, dass nur die wissenschaftliche „externe Evidenz" gewertet wird und nicht, wie von Sackett und der Cochrance Collaboration gefordert, auch die zweite und dritte Säule, nämlich die „klinische Erfahrung“ und die „Bedürfnisse der Patienten“, berücksichtigt werden. Sackett, einer der Gründer der EBM, publizierte bereits 1996 wie die drei Säulen der EBM zusammen, die Wertigkeit der jeweiligen Therapiemethode bilden und dass der gewissenhafte Einsatz von EBM eher zu Mehrkosten führen kann als zu sparen. Wenn, aus welchen Gründen immer, noch keine hohe externe Evidenz aus randomisierten kontrollierten Studien für einzelne Therapie-Methoden vorhanden ist, sollte auf die nächst niedrigere Evidenzstufe zurückgegriffen werden. Keinesfalls dürfen jedoch die klinische Erfahrung und das Wohl des Patienten als wesentliche Entscheidungskriterien vergessen werden! Letztendlich entscheidet nach Sackett, der behandelnde Arzt, welche Wertigkeit der wissenschaftlichen Literatur für die individuelle Behandlung des Patienten zugemessen wird. Diese Ausgabe enthält wissenschaftliche Studien, die das Wissen über Molekularbiologie und Physiologie der Muskulatur erweitern. Allen zugrunde liegt ihr evidenzbasierter Ansatz und die Bedeutung als Translationale Medizin für die Physikalische Medizin. Gerta Vrbová zeigt eine kurze Einführung in die historischen Arbeiten von AV Hill, welche zu dem heutigen Grundverständnis über Muskelkontraktionen wesentlich beigetragen haben. Seine Entdeckungen haben geholfen das Problem wie chemische Reaktionen die Energiebereitstellung für die mechanische Arbeit des menschlichen Muskels bewerkstelligen zu lösen. Hill lehrte biologische Phänomene mathematisch zu ergründen. Eine Basis für die heutige evidenzbasierte Medizin. Die Zellskelett und die L-Type Ca2+ Kanäle welche den Ca2+-Einstrom während der passiven Dehnung der Muskelfaser regulieren wurden von Fabio Francini und Roberta Squecco untersucht. Zusammen mit den elektronenmikroskopischen Ergebnissen von Anna Jakubiec-Puka über die Effekte passiver und aktiver Bewegung des Rattenmuskels bieten sie eine Basis für unsere klinische Arbeit. Sie erklären wie komplett denervierte oder gelähmte Patienten von physikalischen Behandlungen profitieren können und sind aussagekräftige Beispiele für Translationale Medizin. Bei Kindern mit Plexusparese, in Conus-Cauda Patienten und Patienten mit peripheren Nervenläsionen können wir der fortschreitenden Atrophie denervierter/gelähmter Muskelfasern durch passives Bewegen entgegenwirken. Die passiven Bewegungen in zehn Serien zu je 20-30 Wiederholungen, gesamt 200 bis 300 Wiederholungen pro Tag, erhöhen den Ca2+-Einstrom aller gelähmten Muskelgruppen. Die Beiträge von Milan Sedliak et al. und Nejc Sarabon et al. konzentrieren sich auf die neuromuskuläre Ansteuerung bei Senioren. Erstere entwickelten und testeten die Reliabilität eines neuen Testverfahrens um die Haltungsschwankungen im aufrechten Stand zu erfassen. Die zweite Gruppe untersuchte die Auswirkungen von Krafttraining auf die statische Balance. Eine bessere Geschicklichkeit kann Senioren vor Stürzen und deren potenziellen Konsequenzen wie Hüft-, Oberschenkelhals-, Becken- und Beinfrakturen schützen. Ein technischer Beitrag hinsichtlich drahtloser Datenübertragung bei Implantaten von Lukas Kneisz et al. zeigt den Einfluss von isolierenden Schichten auf die Übertragungsantennen. Die Autoren simulierten die Verstimmungseffekte von biologischem Gewebe und schlossen daraus, dass Antennen die mit einem gewebeequivalentem Material umschlossen sind, eine sicherere Übertragung der Messdaten aus dem Körper zu einem externen Empfänger ermöglichen. Dies ist eine wichtige Entwicklung für zukünftige Implantate und technische Hilfen in der Physikalischen Medizin. Ein weiteres Beispiel für EBM-Arbeit in der Physikalischen Medizin ist der Review von Asami Naka et al. Diese Arbeit ist ein wichtiger klinischer Überblick über die analgetische Wirkung von TENS bei verschiedenen Schmerzindikationen in der vorhandenen Literatur (2007-2012). Auch zeigt sie die Effekte und Wirkung der schmerzlindernden Elektrostimulation. Ein großes Problem bei Senioren ist der zunehmende Funktions- und Kraftverlust. Wir haben versucht eine Verbesserung der Kraft und Funktion mit funktioneller Elektrostimulation zu erreichen. Wir konnten demonstrieren, dass mittels funktioneller Elektrostimulation eine deutliche Verbesserung der Funktion, der Histologie als auch der molekularbiologischen Marker in der Muskulatur bei 70-jährigen Senioren erzielt werden kann.

Sackett DL, Rosenberg WM, Gray JA, Haynes RB, Richardson WS. Evidence based medicine: what it is and what it isn't. BMJ. 1996; 312(7023): 71-72.

Jakubiec-Puka A, Sławińska U, Rędowicz MJ, et al. Beneficial effect of locomotor training on the structure of the denervated rat soleus muscle. Basic Appl Myol 2007; 17: 125-127.

Helmut Kern, MD, PhD, Editor-Translational Myology, E-mail: wil.pys.kern-forschung@wienkav.at 